\title{
Escenas de cine y televisión, un recurso didáctico para la enseñanza- aprendizaje de las matemáticas
}

\author{
Cinema and television scenes, a didactic resource for the \\ teaching-learning of the mathematics
}

\section{ALBERTO ZAPATERA \\ Universidad Cardenal Herrera-CEU}

\section{RESUMEN}

El cine y la televisión son recursos didácticos muy interesantes para el diseño y el desarrollo de procesos de enseñanza-aprendizaje. Este trabajo presenta un proyecto para fomentar la utilización de escenas de cine y televisión en las clases de matemáticas, en la que participaron alumnos del Grado de Maestro de Educación Primaria. El proyecto tuvo una gran aceptación por parte de los alumnos, que la consideraron muy motivadora y útil para su futuro profesional. Como resultado del proyecto se creó un "banco de escenas" de películas de cine y series de televisión relacionadas, de forma más o menos explícita, con las matemáticas y se consensuó una lista de recomendaciones para su utilización en las clases de matemáticas.

\section{ABSTRACT}

Cinema and television are very interesting didactic resources for the design and development of teaching-learning processes. This work presents a project to encourage the use of film and television scenes in the classes of mathematics, in this project participated students of the Degree in Primary Teacher. The project had a great acceptance on the part of the students, who considered it very motivating and useful for their professional future. As a result of the project, a "scene bank" of film and television series related, more or less explicitly, with mathematics was created and list of recommendations were agreed for his utilization in the classes of mathematics. un recurso didáctico para la enseñanza-aprendizaje de las matemáticas. EA, Escuela Abierta, 21, 25-45. doi: 10.29257/EA21.2018.03
Recibido: 05/12/2017

Aceptado: 18/05/2018

\section{PALABRAS CLAVES}

Enseñanza de las matemáticas, escenas de cine, recurso didáctico, formación profesores

\section{KEYWORDS}

Mathematic Teaching, Movie Scenes, Didactic Resource, Teacher Training 


\section{INTRODUCCIÓN}

El cambio metodológico impulsado por las orientaciones del Espacio Europeo de Educación Superior (EEES) supone un nuevo desafío para el profesor, que cede su protagonismo al alumno y pasa de mero transmisor de conocimientos a inductor, mediador y guía. Para hacer posible este cambio los profesores deben utilizar distintas estrategias y metodologías y, en este contexto, se incluye la utilización de todo tipo de recursos, incluidos los audiovisuales.

En una sociedad en la que los estudiantes están sometidos a continuos estímulos audiovisuales, los medios audiovisuales en general, y el cine y la televisión en particular, pueden convertirse en herramientas didácticas muy interesantes. Sin embargo, la utilización en las aulas del cine y de la televisión no es una práctica generalizada debido a la falta de infraestructura de algunos centros, a la insuficiente formación de los profesores o a que su utilización supone un cambio profundo en los modelos de enseñanza (Beltrán-Pellicer, 2015); de esta forma, con frecuencia su utilización queda reducida en algunos centros a la proyección indiscriminada de películas sin ninguna planificación ni conexión con los contenidos a impartir.

Aunque existen pocas investigaciones sobre el uso del cine y la televisión como recurso didáctico, hay numerosas recopilaciones de escenas para utilizar en clase de matemáticas, como las páginas web Math and the Movies (Roberts y Roberts, 2014), Mathematical Fiction Homepage (Kasman, 2014) o MMDB of the Mathematical Movi Database (Polster y Ross, 2008).

En España, divulgadores matemáticos como Sorando (2017) con la página Matemáticas en el cine y en las series de TV: un recurso para el aula, el grupo Cinemat (Raga, Muedra y Requena, 2009) con el DVD Matemáticas de cine o Población (2006) con el libro Las matemáticas en el cine, promocionan la utilización de escenas de películas de cine y de series de televisión en las clases de matemáticas.

En esta experiencia, en la que participaron estudiantes para maestro, se proponía fomentar el uso de escenas de películas de cine y series de televisión en el aula de matemáticas; de esta forma, los objetivos de la experiencia, además de motivar a los alumnos participantes, fomentar el trabajo en equipo y generar y consolidar conocimiento matemático, eran:

- Proporcionar a los alumnos del Grado de Maestro de Educación Primaria estrategias y experiencias para utilizar en su futuro profesional.

- Incorporar el uso de las tecnologías de la información y la comunicación (TIC) en las clases de matemáticas.

- Elaborar una serie de recomendaciones para la utilización de escenas de cine y televisión en clase de matemáticas.

- Recopilar escenas de cine y televisión con referencias matemáticas para formar "un banco de escenas" (Anexo 1). 


\section{FUNDAMENTACIÓN}

\section{1. Fundamentación didáctica}

Numerosas películas de cine y series de televisión contienen una gran cantidad de referencias matemáticas, con una característica especial de cara a su utilización en clase: son a-didácticas; es decir, están concebidas para entretener, por lo que su objetivo no es didáctico. Este carácter de entretenimiento y a-didáctico hace que a los alumnos les resulten más interesantes las películas de cine y series de televisión que documentales didácticos.

Sin embargo, ese carácter a-didáctico de las películas de cine y series de televisión supone un reto para el maestro que, cuando decide utilizar una escena como recurso didáctico en la clase de matemáticas, debe realizar una transposición didáctica (Chevallard, 1985) de la escena seleccionada. En este proceso, el maestro, tras identificar el saber matemático determinado que contiene la escena, que estará entre el saber erudito y el saber a enseñar, adaptará ese saber a las características del contexto para que el alumno construya su propio saber (Figura 1).

Figura 1

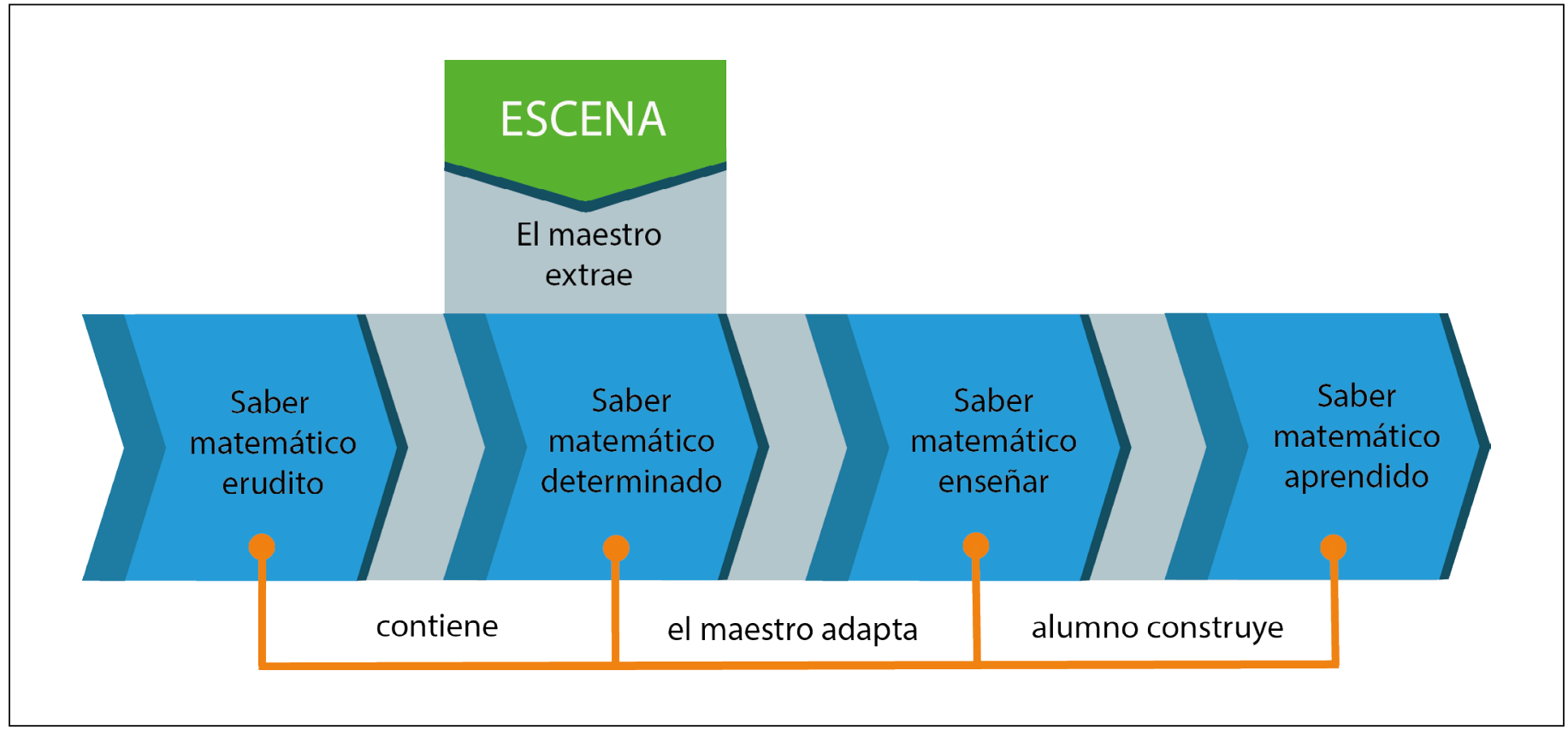

En el proceso de utilización de las escenas como resultado de la transposición didáctica, Beltrán-Pellicer y Asti (2014) proponen un patrón de trabajo que muchos maestros han adaptado en sus experiencias. Este patrón consta de cuatro fases: en la primera fase se proyecta la escena y se plantean las actividades; en la segunda fase los alumnos trabajan en grupos y resuelven las actividades; en la tercera fase se discuten las soluciones propuestas por los grupos; y en la cuarta fase el maestro comenta las soluciones aportadas y describe la solución (Figura 2). 


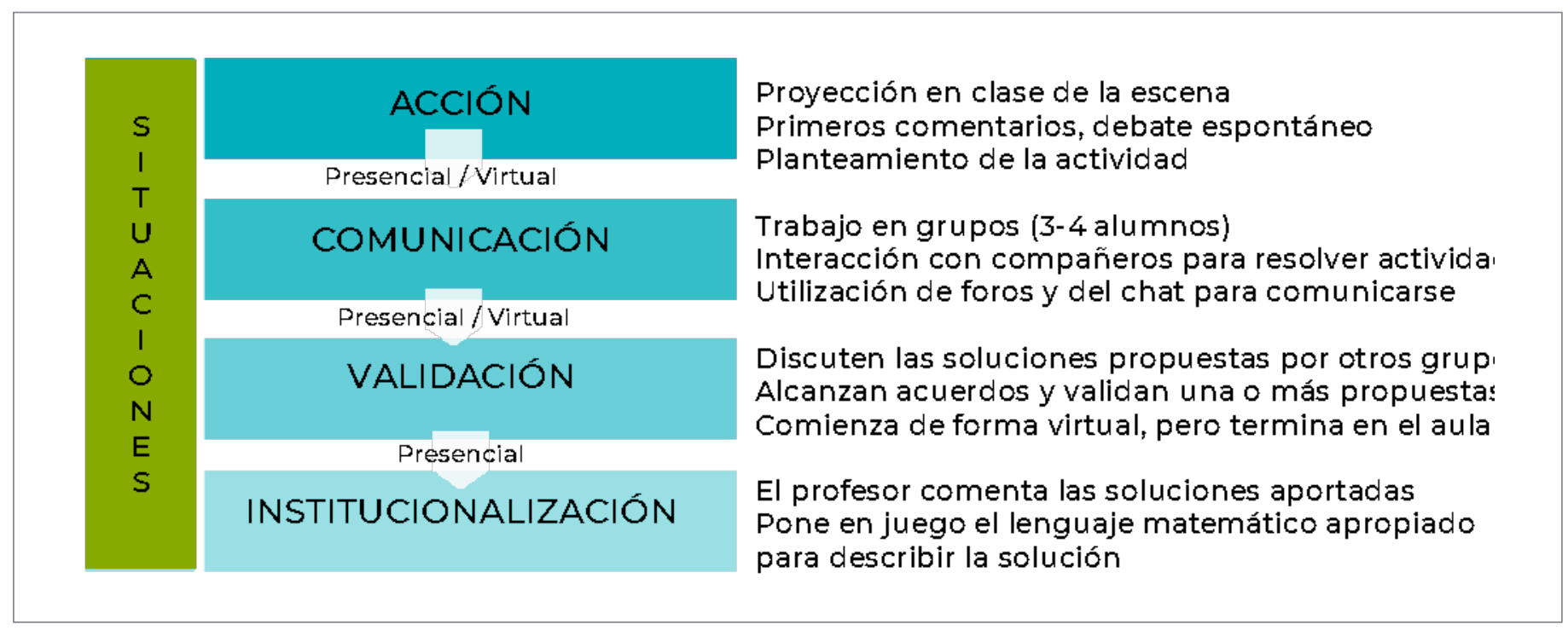

\section{2. Clasificación de escenas}

Las escenas de películas de cine o de series de televisión con referencias matemáticas se clasifican en tres grupos: escenas con gazapos o errores matemáticos, escenas que implican la resolución de un problema y escenas que ponen al descubierto un determinado contexto social (Sorando, 2007; Beltrán-Pellicer y Asti, 2014) (Figura 3).

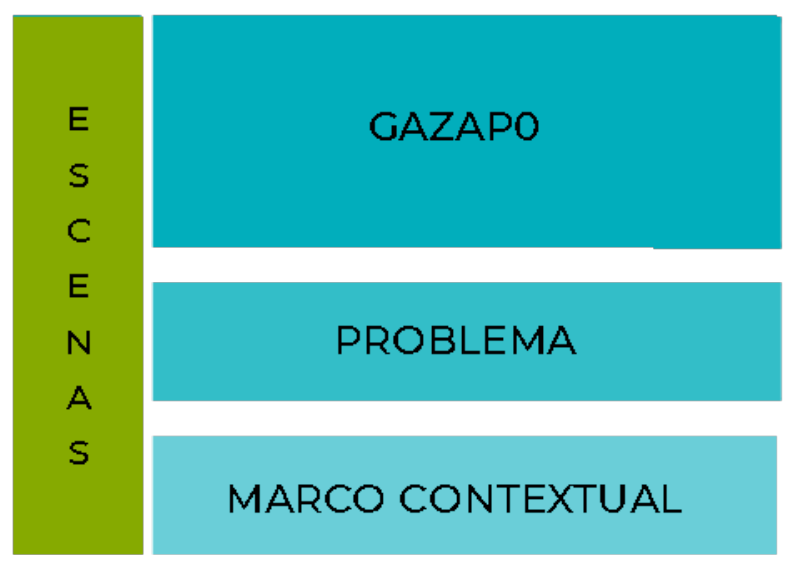

\begin{tabular}{|l|}
\hline De situación \\
\hline De concepto \\
\hline De cálculo \\
\hline De razonamiento \\
\hline Explícito \\
\hline Implícito \\
\hline
\end{tabular}




\subsubsection{Escenas con gazapos}

Los gazapos, o errores matemáticos, que aparecen en las escenas de películas de cine o de series de televisión pueden ser gazapos de situación, de concepto, de cálculo o de razonamiento.

En los gazapos de situación el error aparece en objetos que forman parte del escenario, como una pizarra en una clase o un grafiti en una pared. En un capítulo de los Simpson, Homer al cubo, Homer pasa de su "mundo plano" a la tercera dimensión donde aparece un gazapo de situación que aparentemente contradice el Último Teorema de Fermat, que afirma que no existe ningún número entero positivo, n, mayor que 2 que satisfaga la igualdad $a^{n}+b^{n}=c^{n}$.

En la escena Homer pasea sobre una trama tridimensional y al fondo se observa:

$$
1782^{12}+1841^{12}=1922^{12}
$$

Si se realizan las operaciones con una calculadora de 10 dígitos se obtienen dos expresiones aparentemente iguales:

$$
1782^{12}+1841^{12}=2,541210259 \cdot 10^{39} \quad 1922^{12}=2,541210259 \cdot 10^{39}
$$

Pero si se realizan los cálculos con más todas las cifras se obtienen dos cantidades distintas:

$$
\begin{gathered}
1782^{12}+1841^{12}=2541210258614589176288669958142428526657 \\
1922^{12}=2541210259314801410819278649643651567616
\end{gathered}
$$

Los gazapos de concepto son errores matemáticos debidos a la falta de comprensión de algún contenido matemático. Una escena de la película Cortina rasgada, en la que durante la guerra fría un espía norteamericano intenta contactar con una organización secreta llamada "pi", contiene un gazapo de concepto: un agente de contraespionaje intenta desenmascarar al espía norteamericano y define $\pi$ de forma incorrecta.

- Tal vez una letra griega, tal vez $\pi$. Matemáticas. $\pi$ es el radio de la circunferencia de un círculo por su diámetro. ¿No es cierto?

- Sí, usted es un hombre muy instruido

- Fui a la escuela nocturna. Acudí a una escuela nocturna especial y nos dijeron todo sobre $\pi$

Esta definición de $\pi$ contiene varios gazapos de concepto al mezclar de forma arbitraria varios términos matemáticos.

Los gazapos de cálculo son errores en la realización de operaciones y son muy frecuentes en las películas. Muchas veces los gazapos de cálculo se producen de forma inconsciente, como en la película Sal gorda, que narra la crisis creativa que un famoso compositor. En la escena seleccionada, el mánager le ofrece una última oportunidad para salvar su carrera y comete un error de cálculo cuando advierte que:

- Tenéis 47 horas para hacer 10 canciones, es decir, 4 horas y 7 minutos por canción; eso si no dormís.

Realizando correctamente los cálculos serían 4 horas y 42 minutos por cada canción. 
En los gazapos de razonamiento los protagonistas realizan razonamientos matemáticos de forma errónea. En la película Stargate, un científico que trabaja para el ejército asocia los 7 símbolos encontrados en unas excavaciones con localizadores que marcan una ruta en el espacio.

- Siete puntos que trazan una ruta hacia un lugar determinado; para encontrar el destino en cualquier espacio tridimensional necesitamos 6 puntos que determinen una localización exacta (dibuja un punto en cada una de las caras de un cubo y los une de dos en dos marcando la intersección de los tres segmentos)

- Ha dicho que necesitaba 7 puntos

- Bueno, no, 6 para el destino, pero para trazar una ruta se necesita un punto de origen (dibuja otro punto en el exterior del cubo y lo une con el marcado anteriormente).

El razonamiento que sigue el científico parece adecuado, pero... ipara localizar cualquier punto en un espacio tridimensional solo se necesitan tres coordenadas!

\subsubsection{Escenas con problemas matemáticos}

Algunas escenas plantean problemas matemáticos, entendidos en este caso como ejercicios en los que se describen situaciones tomadas de la vida real. En algunas escenas los problemas se plantean de forma explícita, mientras que en otras se plantean de forma implícita y el profesor debe explicitarlos.

Un problema explícito se presenta en la película El florido pensil, que recrea el sistema educativo de la posguerra española. En la escena se plantea el siguiente problema: "En un cesto hay 36584 huevos, ¿cuántos pares de huevos contiene?". Varios alumnos contestan que 18292 pares de huevos, hasta que un alumno hace uso del sentido común y pone en evidencia lo absurdo que resultan muchos problemas que se plantean en las clases de matemáticas.

- Es imposible, por los huevos de abajo

- ¿Qué es eso de los huevos de abajo?

- Pues eso, que 38584 huevos son muchísimos huevos y no hay cesto para tantos huevos, y si los hubiera, los de abajo se aplastarían. ¡Buf, qué asco! ¡Todo el cesto chorreando de huevos aplastados! ¡Qué horror!

Un problema implícito aparece en la película La fórmula preferida del profesor que narra cómo un profesor que, a consecuencia de un accidente tenía una memoria limitada a los 80 últimos minutos, inculcó su amor por las matemáticas a un niño. En la escena unos alumnos comentan el valor del número $\pi$.

- $\pi$ es igual a 3,141592653

- ¡Qué lástima! ¿Por qué no lo dejarían en 3?

- Si lo dejas en 3, te saldría un hexágono en lugar de un círculo

El profesor debe explicitar el problema, por ejemplo de la siguiente forma:

Si el valor de $\pi$ fuera 3, ¿cuánto mediría la longitud de una circunferencia en función de su radio? ¿Y el área del círculo? ¿Cuánto mide el perímetro del hexágono regular inscrito en la misma circunferencia? ¿Y su área? Compara los resultados" 


\subsubsection{Escenas que muestran un marco contextual}

Con frecuencia el gazapo o el problema matemático que presenta una escena solo es un pretexto para poner en evidencia un determinado aspecto de la sociedad. En La vida es bella, un judío italiano emplea la imaginación para proteger a su pequeño hijo de los horrores de un campo de concentración nazi. En la escena seleccionada la directora de una escuela plantea un problema, aparentemente matemático, que descubre una sociedad sometida al nazismo.

- En el tercer grado, ifijaos qué problema les pusieron! Me acuerdo porque me impresionó: "Un demente cuesta al estado 4 marcos diarios, un mutilado 4 marcos y medio, un epiléptico 3 marcos y medio. Visto que la cuota media es de 4 marcos diarios y que los pacientes son 300.000, ¿cuánto se ahorraría el Estado si estos individuos fueran eliminados, suprimidos?"

- ¡Dios mío, no es posible!

- Ésa es la reacción que tuve yo, Dora: ¡Dios mío, no es posible! No es posible que un pequeño de 7 años resuelva un problema de este género. Es un cálculo complejo, con proporciones, con porcentajes. Se requieren unas nociones mínimas de álgebra; es un problema de Escuela Superior.

\section{DESARROLLO DE LA EXPERIENCIA}

\subsection{Participantes}

En la experiencia participaron 45 estudiantes para maestro de la asignatura "Aprendizaje y Didáctica de las Matemáticas en Educación Primaria" del $3^{\circ}$ curso del Grado de Maestro de Educación Primaria. Para la realización de las actividades se dividieron en 11 grupos de 3, 4 o 5 estudiantes.

\subsection{Etapas}

La experiencia se desarrolló en cuatro etapas (Figura 4) durante el segundo cuatrimestre del curso 2016-17.

Figura 4

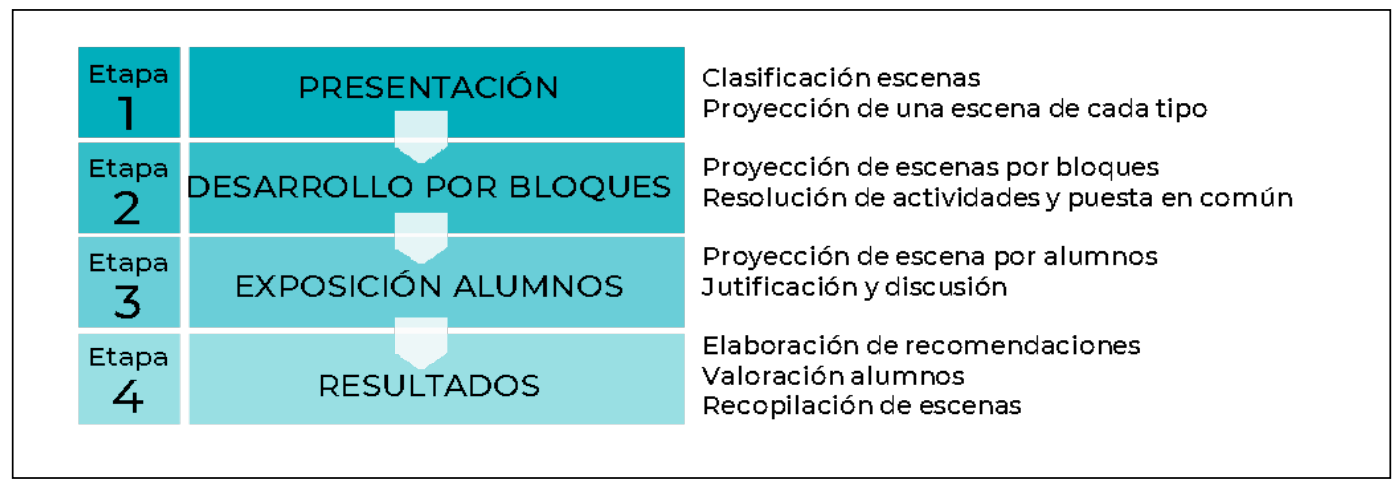




\subsubsection{Etapa 1. Presentación de la experiencia}

En la primera etapa se informó a los alumnos sobre el desarrollo de la experiencia y sus objetivos, se mostró la clasificación de escenas y se proyectó y comentó una escena de cada uno de los tipos.

\subsubsection{Etapa 2: Desarrollo del proyecto por bloques}

En la segunda etapa, el profesor seleccionó y proyectó una escena con referencias matemáticas de cada bloque de la asignatura "Aprendizaje y Didáctica de las Matemáticas", que se corresponden aproximadamente con los bloques del currículo de Matemáticas de Educación Primaria (R.D. 126/2014) (Tabla 1).

\section{Tabla 1}

Bloques de la asignatura y del currículo de Primaria

\begin{tabular}{|c|c|c|}
\hline OQUE & “APRENDIZAJE y DidÁctica DE LAS Matemáticas" & Currículo Primaria \\
\hline 1 & La enseñanza-aprendizaje de las matemáticas en Educación Primaria & $\begin{array}{l}\text { Procesos, métodos y ac- } \\
\text { titudes en matemáticas }\end{array}$ \\
\hline 2 & Números y operaciones y su didáctica en Educación Primaria & Números \\
\hline 3 & La medida: estimación y cálculo y su didáctica en Educación Primaria & Medida \\
\hline 4 & Geometría y su didáctica en Educación Primaria & Geometría \\
\hline 5 & $\begin{array}{l}\text { Tratamiento de la información, azar y probabilidad y su didáctica en } \\
\text { Educación Primaria }\end{array}$ & $\begin{array}{l}\text { Estadística y probabili- } \\
\text { dad }\end{array}$ \\
\hline
\end{tabular}

Las escenas seleccionadas se usaron como presentación, motivación e hilo conductor de cada bloque. Después de la proyección, los alumnos debatieron por grupos reducidos la escena, realizaron las actividades de la tabla 2 y, posteriormente, se realizó la puesta en común.

\section{Tabla 2}

\section{Bloque ACTIVIDAD}

1 Expresa tu opinión sobre la escena

2 Explica algún detalle de la escena que te haya llamado la atención

3 Una actividad específica sobre algún aspecto concreto de la escena

Busca alguna escena de películas o series de televisión relacionadas con el bloque y explícalas brevemente 
Para realizar la cuarta actividad de cada bloque los grupos de alumnos realizaron una búsqueda libre según sus propios criterios y medios durante dos semanas por bloque.

\section{Bloque 1. La enseñanza-aprendizaje de las matemáticas en E. P.}

En este bloque se proyectó el cortometraje 3 minutos y 14 segundos realizado por un grupo de estudiantes de Comunicación Audiovisual de la Universidad Autónoma de Barcelona para celebrar el centenario de la Real Sociedad Matemática Española, que muestra los aspectos más cotidianos del día a día de una adolescente con un denominador común: las matemáticas.

Los números son los que te despiertan cada mañana aunque no quieras despertar. Son los que te recuerdan que llegas tarde al autobús. Son tu nómina, tus facturas, tus preocupaciones. Son una cita, tu alquiler, son tu moneda. Son la distancia recorrida y también la que queda por recorrer. Son la temperatura de un día soleado. Son el ritmo de la vida. Son la simetría de una flor, son las ondas de tu pelo. Son el peso de una manzana [...]. Las matemáticas nos rodean, están ahí, aunque no las vea; igual que la vida los números nos presentan dificultades pero también nos enseñan que no existe un problema sin solución. La vida son matemáticas: vive y vívelas.

A la mayoría de los alumnos el cortometraje les pareció muy interesante al mostrar la gran cantidad de aspectos de la vida diaria en la que intervienen las matemáticas.

Los grupos destacaron diferentes aspectos del cortometraje que muestran cómo las matemáticas no solo están en las clases y describieron y comentaron muchos aspectos matemáticos de la vida diaria que no aparecían en el cortometraje.

Los estudiantes localizaron y clasificaron 24 escenas relacionadas con este bloque.

\section{Bloque 2. Números y operaciones y su didáctica en E. P.}

La escena seleccionada se titula Una clase de matemáticas y pertenece al show de Abbott y Costello, un dúo cómico muy popular en EEUU que rodaron en 1952 una serie de televisión sobre la vida de dos parados. En la escena Costello intenta engañar a su casero para pagarle solo 28 dólares por el alquiler de 13 semanas a 7 dólares la semana. Para ello Costello demuestra que $13 \times 7$ es igual a 28 de tres maneras distintas: haciendo la división 28/7, multiplicando 13 x 7 y sumando directamente 7 veces 13 . La fuente de confusión está en ignorar el valor posicional de las cifras, mezclando decenas con unidades.

- Escribo el 7 y luego voy a dividir 28 entre 7 y escribo 28 aquí. Procedamos: 2 dividido entre 7 [...] pondré el 2 aquí y 21 entre 7 a 3, así que pondré 3 aquí, así que 28 entre 7 es 13

- Pongo el 13, ahora por 7, 7 multiplicado por 13 deberá dar 28, ¿cuántos son 7 por 3? 21, ¿cuántos son 7 por 1? 7, ¿7 más 1 ? 8 y el 2 que tenemos aquí, 28.

- ¿Quiere que escriba 7 veces el 13? Aquí está: 3, 6, 9, 12, 18, 21, 22, 23, 24, 25, 26, 27 y 28

A todos les pareció muy divertida la escena y muchos señalaron la importancia de las matemáticas para no ser engañados. 
Los grupos indicaron detalles muy diversos de la escena, desde la ingenuidad del casero a la astucia del inquilino, pero apenas mencionaron aspectos matemáticos.

Todos los grupos comentaron que los errores se deben al orden, a la posición de las cifras y alguno mencionó que mezcla unidades y decenas, centrándose más en la suma y en la multiplicación que en la división.

Los estudiantes localizaron 57 escenas relacionadas con el tema.

\section{Bloque 3. La medida: estimación y cálculo y su didáctica en E. P.}

La escena proyectada pertenece a la película Factotum, basada en la vida del escritor estadounidense Charles Bukowski, adicto al alcohol y a las apuestas. En la escena, el protagonista realiza unos cálculos para saber la hora con un reloj que adelanta 35 minutos cada hora... jcon gazapo incluido!

- ¡Eh, quiero saber qué hora es! Dijiste que arreglarías el reloj.

- Vale, vamos a ver... Pusimos el reloj en hora, con la tele, anoche a las 12. Sabemos que adelanta 35 minutos cada hora. Marca las $7 y$ media de la tarde. pero sabemos que no puede ser, porque apenas ha oscurecido. Vale. Son 7 horas y media, 7 veces 35 minutos, son 245 minutos, la mitad de 35 son 17 y medio, eso hacen 252 minutos y medio. Bien, entonces restamos 4 horas y 42 minutos y medio, o sea que hay que retrasar el reloj a las 5 y 47. Eso es. Son las 5 y 47 . La hora de cenar y no tenemos nada que comer.

La mayoría opinó que la escena no aporta nada a la película ya que, dicen, se trata simplemente de unos cálculos que no tienen demasiado sentido y que están mal realizados; algunos señalaron el ambiente degradante en el que se mueve el protagonista y pocos se centraron en aspectos matemáticos.

Una forma de resolver el problema sería aplicando la constante de proporcionalidad entre los minutos que marca el reloj y los minutos reales $(\mathrm{k}=95 / 60)$. De esta manera la hora exacta sería las 12:18:57, muy lejos de las 05:47 que dice el protagonista. Aunque reconocen que el protagonista no ha planteado bien el problema y que realiza mal las operaciones, pocos grupos fueron capaces de aplicar el razonamiento proporcional a partir de la constante y, además, cometieron errores como considerar solo 7 horas y media y no 19 y media o equivocarse al transformar los minutos en horas y minutos.

Se localizaron 10 escenas en este bloque.

\section{Bloque 4. Geometría y su didáctica en Educación Primaria}

La escena seleccionada pertenece al capítulo Springfield próspero de la serie de los Simpson, que narra la vida de una familia americana en un pueblo ficticio llamado Springfield. Tres guionistas de la serie son matemáticos, por lo que la serie tiene numerosas alusiones matemáticas. En la escena, Homer encuentra en los aseos las gafas del Secretario de Estado, Kissinger, se las pone y recita el Teorema de Pitágoras.

- ¡Esto no se ve en un excusado todos los días! ¿Alguien perdió sus anteojos? ¿Nadie? ¡Uf! (se pone las gafas). La suma de las raíces cuadradas de dos lados de un triángulo isósceles es igual a la raíz cuadrada del lado restante

- Eso es el equilátero, jidiota! 
Aunque los alumnos han visto muchos capítulos de la serie, reconocieron que no se habían fijado en las continuas referencias matemáticas que contiene.

La mayoría de los grupos, en relación a la escena proyectada, señalaron la relación muy extendida entre gafas e inteligencia.

Todos los grupos señalaron los errores cometidos por Homer al enunciar el Teorema de Pitágoras, centrando sus observaciones en los fallos cometidos: isósceles en lugar de rectángulos y el enredo entre raíces y cuadrados.

Se localizaron 22 escenas en el bloque de geometría.

\section{Bloque 5. Tratamiento de la información, azar y probabilidad y su didáctica en E. P.}

La escena estudiada pertenece a la película Black Jack, en la que el protagonista, para poder pagar la matrícula de la universidad, se une a un grupo de estudiantes que, con su profesor de matemáticas, consigue grandes ganancias jugando al black-jack. En la escena, el profesor de matemáticas presenta en clase el conocido problema de Monty Hall, basado en un concurso de televisión, que en su momento desencadenó un interesante debate entre matemáticos.

Te ofrecen elegir entre tres puertas distintas, ¿de acuerdo? A ver, tras una de estas puertas hay un coche nuevo, tras las otras, dos cabras. ¿Qué puerta escogerías, Ben?

¿La uno?

La uno. Ben escoge la puerta uno. Ahora el presentador, que por cierto sabe lo que hay detrás de todas las puertas decide abrir otra puerta, digamos que elige la número tres, tras la cual aparece juna cabra!. Y ahora, Ben, el presentador va y te dice, "Ben, sigues con la puerta uno o la cambias por la dos?” ¿Te interesa cambiar de puerta?

\section{[...] La mayoría no cambiará de puerta por paranoia, miedo, emociones...}

Varios grupos no acabaron de entender la explicación del concurso y opinan que al quedar dos puertas, cada una tiene un $50 \%$ de probabilidad de contener el coche. Incluso después de estudiar varias resoluciones diferentes, algunos estudiantes seguían sin entender por qué es preferible cambiar de puerta.

Aunque a algunos grupos les ha llamado la atención ciertos términos matemáticos y la disyuntiva entre "matemáticas y emociones", la mayoría se ha centrado en aspectos más superficiales como la pizarra o la disposición de los alumnos.

Se localizaron y clasificaron 16 escenas de este bloque.

\subsubsection{Etapa 3: Exposición de alumnos}

En la tercera etapa, los 11 grupos de alumnos localizaron escenas relacionadas con un tema matemático propuesto por el profesor; después, en el aula, presentaron, proyectaron y justificaron una de las escenas a sus compañeros de clase y moderaron el correspondiente debate.

A continuación se muestran y comentan brevemente las escenas presentadas por los distintos grupos. 
El grupo 1 presentó una escena del cortometraje Binta y la gran idea que muestra la injusticia en la sociedad y la necesidad de asistir a la escuela. En la escena Binta, una niña senegalesa de 7 años, narra cómo un comerciante desaprensivo engaña a su tía.

Falu es mi tía, la madre de Soda. Cuando no está enferma ella trabaja recogiendo fruta que vende a los comerciantes que vienen de la ciudad

- Vamos a ver lo que tiene aquí. ¿Solo tienes estas naranjas?¿A cuánto el kilo?

- A 400

- [...] Te doy 200

$-¿ 200$ ?

- Depende de los kilos

- Tengo dos cubos

- ¿De 10 kilos cada uno?

- Eso es

- O sea, 8 kilos en total. Asís es que son 8 kilos a 200... Te voy a dar 1100, jeso es lo justo! Mira, te doy dos billetes de 400 para no dejarte sin cambio.

Mi tía nunca fue a la escuela

\section{Grupo 2. Números naturales}

La película Midiendo el mundo, que se desarrolla en la Alemania del siglo XIX, narra la vida de dos científicos alemanes: el matemático Gauss y el geógrafo y naturalista Humboldt. En la escena el maestro castiga a los alumnos a sumar los números naturales del 1 al 100; el niño Gauss, con 9 años, consigue sumarlos rápidamente.

- ¿De dónde ha sacado esto?

- Bueno, nos ha pedido que sumáramos todos los números del 1 al 100. 1 más 100 da 101, 2 más 99 da 101, 3 más 98 da 101... jsiempre da 101! Y eso se puede hacer 50 veces y 50 por 101 da 5050.

- Gauss, jal rincón! En silencio y te quedas hasta el final.

\section{Grupo 3. Fracciones}

En Granujas de medio pelo cuatro ladrones planean robar un banco cavando un túnel desde una tienda de galletas hasta un banco. En la escena discuten cómo repartirse el botín entre ellos cuatro y la vendedora de galletas.

- Bueno, ¿sabéis qué? Que (Frenchy) cobre una parte, pero no una parte entera.

- ¿Qué tal si todos cobramos un cuarto y ella, digamos, un tercio?

- ¡Tú estás chinao! Entonces cobraría más que nosotros.

- ¿Cómo lo sabes?

- Además, ¿de dónde sacas cuatro cuartos y un tercio? ¿No sabes sumar?

- Mira, yo en quebrados no me meto, ¿vale? 


\section{Grupo 4. Potencias y raices}

En la película Cadena de favores, el profesor de sociales propone a sus alumnos que piensen una idea para hacer del mundo un sitio mejor. A Trevor, un niño de 11 años, se le ocurre hacer tres favores y que los beneficiados hagan otros tres favores a otras tres personas y así sucesivamente creando una cadena de favores.

- Piensa una idea para cambiar el mundo y ponla en acción

$-[\ldots]$

- Soy yo y son tres personas. Y yo voy a ayudarlas, pero tiene que ser con algo grande, con algo que no puedan hacer solas. Lo haré por ellas y ellas lo harán por otras tres. Son nueve y yo hago tres más.

\section{Grupo 5. Proporcionalidad y porcentajes}

En una escena del El bazar de las sorpresas, un vendedor ofrece un interesante descuento a una clienta... ¡ con gazapo incluido!

- ¿Cree que sería posible obtener un pequeño descuento?

- ¡Oh, sí! Estamos en plenas rebajas de verano y todo tiene un 25\% de descuento. Aquí tenemos, por ejemplo, esta polvera: su precio real es 3,90, pero puede adquirirla gracias al descuento por 2,24.

- ¿De verdad? ¡Es una oferta maravillosa!

\section{Grupo 6. Masa y peso}

En Yo hice a Roque III, el dueño de un gimnasio entrena a un amigo boxeador. En la escena los protagonistas se hacen un lío al pasar a kilogramos las 135 libras que pesa Roque.

- ¿Qué pasa? ¿He adelgazado mucho?

- No sé, aquí pone 135

- ¿Kilos? ¿Cómo voy a pesar 135 kilos?

- No son kilos, son libras, es que esta báscula es inglesa

- ¡Ah! ¿Y cuántos kilos son 135 libras?

- Espera que ahora viene Paco con la calculadora.

\section{Grupo 7. Capacidad y volumen}

En La jungla de cristal 3 un policía y un electricista deberán superar una serie de pruebas para desactivar las bombas que un terrorista está colocando por la ciudad. Una de las pruebas es el conocido problema de las garrafas.

- Debe haber dos garrafas en la fuente, una de 5 galones y otra de 3 galones. Llene una de ellas con 4 galones justos de agua y póngala sobre la báscula y el contador (de la bomba) se apagará. Sea exacto: una onza de más o de menos provocará la detonación. Si sigue vivo dentro de 5 minutos, volveremos a hablar

- ¡Espere!, jespere! [...]

- ¡Lo tengo!, jlo tengo! Aquí hay 2 galones justos (señala la garrafa de 3) ¿no?, lo cual deja un galón exacto de espacio libre ¿verdad'. Esta (señala la garrafa de 5) se llena con 5 galones, ¿no? Pasas 1 galón de los 5 a esta y nos quedan ;4galones! 


\section{Grupo 8. Geometría plana}

En Las diabólicas, el director de un internado convive con su esposa y su amante; las dos mujeres, cansadas de sufrir los malos tratos del hombre, deciden acabar con él. La atmósfera obsesiva de la escena, que refleja la angustia de la profesora, deja en un segundo término la tarea matemática: la superficie del hexágono regular inscrito en una circunferencia.

- ¿Qué les pasa? ¿Por qué me miran todos? ¿Qué tengo? Continúe, superficie del hexágono conociendo el radio de la circunferencia. Vamos, estoy esperando. Estoy esperando

- $6 A B$ (lado del hexágono) por $\mathrm{OH}$ (radio de la circunferencia) partido por 2

- Gracias, muy bien, vuelva a su sitio

\section{Grupo 9. Geometría espacial}

En el capítulo de los Simpson Las chicas solo quieren sumar, Lisa quiere resolver problemas y realizar cálculos como hacen los chicos y se rebela contra la separación por género que le impide aprender matemáticas. En la escena el maestro pide a los alumnos hallar el volumen de un muñeco de nieve formado por tres esferas... iy una zanahoria!

- Ahora niños, ¿quién puede decirme el volumen de este muñeco de nieve? ¿Alguien?

- Solo añada el volumen de las esferas, sabemos los radios

- Olvidó el volumen de la nariz de zanahoria: un tercio de la base por su altura. ¡Oh, matemáticas! ¡Las extrañaba!

\section{Grupo 10. Estadística}

En Ciudad mágica, una empresa que se dedica a los sondeos electorales está al borde de la bancarrota, descubre por casualidad que los resultados que se obtienen en una pequeña ciudad son exactamente iguales a los que se obtienen en todos los Estados Unidos. Este descubrimiento le permitirá ofrecer sondeos muy fiables con un pequeño coste y una alta fiabilidad.

- Schinger encuestó a miles de personas de todo el país y Hupendecker solo encuestó a unas pocas de un pequeño pueblo. Mira los resultados, fíjate, 69 y 69. ¡Son idénticos! Estoy seguro, esta podría ser la solución. Escucha, piensa en esto: un pueblo que piensa exactamente igual que toda una nación junta.

- Tú estás hablando de una utopía

\section{Grupo 11. Probabilidad}

En Justiercia, un parado y un pensionista, cansados de la situación social provocada por la crisis económica, deciden convertirse en justicieros. En la escena interrogan a un político al que le ha tocado siete veces la lotería. La escena incluye un gazapo de cálculo... o de razonamiento. 
- Te compras un décimo de la lotería nacional y como toca siempre... ¿Cuántas veces dices que te ha tocado la lotería? ¿cuántas? ¡Siete!, ¿siete? Joder, te has comido al calvo de la lotería de navidad. ¿Sabes cuál es la probabilidad de que te toque el gordo? Una entre 16 millones, 116 millones!... que multiplicado por 7 , eso hace...!un cojón!

\subsubsection{Etapa 4: Resultados}

En esta etapa, y después de las proyecciones y exposiciones de los alumnos, se estableció un debate sobre la experiencia y se consensuaron las siguientes recomendaciones para utilizar escenas de películas de cine y de series de televisión en clase de matemáticas:

1. Utilizar escenas de corta duración donde se concentren las referencias matemáticas. (Las películas completas son muy largas, se necesitarían dos o más sesiones y se dispersaría la atención del alumno).

2. Seleccionar las escenas y actividades en función del conocimiento matemático de los alumnos

3. Programar los objetivos y la forma de conseguirlos: motivar al inicio de un tema, repasar al final del tema, apoyar o reforzar un determinado concepto, proponer problemas, localizar gazapos...

4. Antes de la proyección de la escena presentar un breve resumen del argumento de la película.

5. Después del visionado proponer a los alumnos actividades verbales y escritas para conseguir el objetivo propuesto.

Al final del proyecto se pasó a los alumnos una encuesta para que valoraran el proyecto y su utilización en su futuro profesional. En la encuesta debian valorar, en una escala de 1 a 4, el interés, la eficacia, la conveniencia de extenderlo a otras asignaturas y la utilidad del proyecto en su futuro profesional (Figura 5).

\section{Figura 5}

\section{ENCUESTA DE VALORACIÓN DEL PROYECTO}

1. Clasifica el Interés del proyecto

$\square$ Sin Interés

Poco Interés

Interesante

Muy Interesante

2. Callfica la eficacla del proyecto

$\square$ Ineficaz

Poco eficaz

$\square$ Eflcaz

Muy eficaz

3. ¿Crees que sería convenlente extender el proyecto a otras aslgnaturas?

$\square$ No convenlente $\square$ Poco convenlente $\square$ Convenlente $\square$ Muy convenlente

4. ¿Cres que el proyecto puede serte útll en tu futuro profeslonal?
$\square$ Inútll
$\square$ Poco útll
$\square$ ÚtIl
Muy útll

Escrlbe las observaclones que creas convenlente: 
Los resultados obtenidos (Tabla 3) muestran una valoración de 3'69 sobre 4. Casi la totalidad de los alumnos calificaron como alto y muy alto los cuatro aspectos considerados, siendo el mejor valorado la utilidad en el futuro.

\section{Tabla 3}

\section{Resultados de la encuesta de valoración}

\begin{tabular}{|c|c|c|c|c|}
\hline & 2 & 3 & 4 & Media \\
\hline Interés & & 11 & 34 & 3'76 \\
\hline Eficacia & & 15 & 30 & $3^{\prime} 67$ \\
\hline Otras asignaturas & 1 & 19 & 25 & $3{ }^{\prime} 53$ \\
\hline Utilidad en el futuro & & 9 & 36 & $3 ’ 80$ \\
\hline Total & 1 & 54 & 125 & $33^{\prime} 69$ \\
\hline
\end{tabular}

Con todas las escenas proyectadas y otras más localizadas por los alumnos a lo largo de la experiencia se creó un "banco" con 125 escenas de películas de cine y de series de televisión con referencias matemáticas (Anexo 1).

\section{CONCLUSIONES E IMPLICACIONES PARA LA ENSEÑANZA}

Al programar esta experiencia para utilizar las escenas de cine y televisión como recurso didáctico en la enseñanza-aprendizaje de matemáticas, además de motivar a los alumnos, fomentar el trabajo en equipo y generar y consolidar conocimiento matemático, se fijaron cuatro objetivos: (1) proporcionar nuevas estrategias y experiencias a los alumnos para su futuro profesional, (2) incorporar el uso de las TIC en la clase de matemáticas, (3) elaborar recomendaciones para utilizar las escenas en clase y (4) formar "un banco de escenas".

Tal y como se comprobó en la encuesta de valoración, los alumnos mostraron un interés y una motivación muy altos durante todo el proyecto; este interés se plasmó en una alta participación e implicación de los alumnos en todas las actividades programadas.

Se fomentó el trabajo en equipo ya que gran parte de este proyecto se realizó en grupos reducidos de alumnos que resolvieron las actividades planteadas y debatieron entre ellos para consensuar la escena a presentar a los compañeros.

Con esta experiencia los alumnos generaron y consolidaron su propio conocimiento matemático: en la selección de escenas debían identificar elementos, conceptos y problemas matemáticos y en la exposición debían presentar, explicar, justificar y argumentar la escena en clase.

La puntuación más alta en la encuesta fue la utilidad para su futuro profesional, con un 3'80 sobre 4; esta valoración nos hace pensar que los participantes utilizarán esta experiencia en sus clases, por lo que consideramos que se ha conseguido el primer objetivo, es decir, proporcionar nuevas estrategias y experiencias a los alumnos para su futuro profesional. 
Para localizar las escenas usaron internet y en clase utilizaron el ordenador y el proyector para exponer a los compañeros las escenas preparadas. De esta manera se consiguió el segundo objetivo: incorporar el uso de las TIC en clase de matemáticas.

Los participantes debatieron extensamente la experiencia y consensuaron unas recomendaciones para facilitar la utilización de escenas en clase de matemáticas, que pueden resultarles de gran utilidad en su futuro profesional. Con ello se cumplió el tercer objetivo: elaborar unas recomendaciones para la utilización de las escenas.

El interés y la participación de los participantes permitieron recopilar y clasificar por su contenido matemático 125 escenas de películas de cine o series de televisión relacionadas, de forma más o menos explícita, con las matemáticas. De esta manera se cumplió también el objetivo de crear un "banco de escenas" con referencias matemáticas.

Aunque esta experiencia se ha llevado a cabo en una asignatura de Didáctica de las Matemáticas, un aspecto muy interesante, y que ha sido altamente valorado por los participantes, es la posibilidad de extenderla a otras asignaturas. Nuestro deseo es que esta experiencia tenga una continuación en próximos cursos, se extienda a otras asignaturas y, especialmente, que sirva de referencia para nuestros alumnos en su futuro profesional.

\section{REFERENCIAS BIBLIOGRÁFICAS}

Beltrán-Pellicer, P. (2015). Series y largometrajes como recurso didáctico en Matemáticas en Educación Secundaria. (Tesis Doctoral), Universidad Nacional de Educación a Distancia (UNED).

Beltrán-Pellicer, P. y Asti, A. (2014). Utilización didáctica del cine en Matemáticas. Enseñanza er Teaching, 32(2), 123. DOI: http://dx.doi.org/10.14201/et2014321123145

Chevallard, Y. (1985). La transposition didactique (Vol. 95). Grenoble, Francia : La Pensée Sauvage.

Kasman, A. (2014). Mathematical Fiction Homepage. Recuperado de http://kasmana.people.cofc.edu/ MATHFICT/

Población Sáez, A. J. (2006). Las Matemáticas en el Cine. Granada: Proyecto Sur de Ediciones.

Polster, B. y Ross, M. (2008). MMDB The Mathematical Movie Database. Recuperado de http://www.qedcat.com/ moviemath/index.html

Raga, M. C., Muedra, A. y Requena, J. (2009). Matemáticas de cine. Valencia: Generalitat Valenciana Conselleria d’Educació.

Real Decreto 126/2014, de 28 de febrero, por el que se establece el currículo básico de la Educación Primaria. Boletín Oficial del Estado. Madrid, 01 de marzo de 2014, núm. 52, p. 19388-19393.

Roberts, F. y Roberts, D. (2014). Math and the Movies! Recuperado de http://mathbits.com/MathBits/MathMovies/MathMovies.htm

Sorando Muzás, J. M. (2007). Gazapos matemáticos en el cine y en la televisión. Suma: Revista sobre Enseñanza y Aprendizaje de las Matemáticas, 55, 117-125

Sorando Muzás, J. M. (2017). Matemáticas en tu mundo: matemáticas en el cine y en las series de TV: un recurso para el aula. Recuperado de http://matematicasmundo.ftp.catedu.es/CINE/cine.htm 


\section{ANEXO I. BANCO DE ESCENAS}

\section{BLOQUE 1. PROCESOS, MÉTODOS Y ACTITUDES EN MATEMÁTICAS_}

\subsection{Las matemáticas en la vida diaria}

$\begin{array}{ll}3 \text { minutos y 14 segundos) } & \text { https://www.youtube.com/watch?v=oWNh1yIzXSs } \\ 3 \text { x } 3 & \text { https://www.youtube.com/watch?v=hk9QRPlbxuo\&feature=youtu.be } \\ \text { El apartamento (1:20-2:40) } & \text { http://www.dailymotion.com/video/xvg6hj_el-apartamento-1960_shortfilms } \\ \text { El manantial de las colinas } & \text { https://vimeo.com/38734536 } \\ \text { Más extraño que la ficción } & \text { https://vimeo.com/86497480 } \\ \text { Moneyball } & \text { https://vimeo.com/45393628 } \\ \text { Pipas } & \text { https://www.youtube.com/watch?v=H1v-bCyeIR4 }\end{array}$

1.2. Clases de matemáticas en distintas épocas

$\begin{array}{lll}8 & \text { Calabuch } & \text { https://www.youtube.com/watch?v=ytVaJDmaTk4 } \\ 9 & \text { Cinema Paradiso } & \text { https://vimeo.com/28243492 } \\ 10 & \begin{array}{l}\text { El enigma de Kaspar } \\ \text { Hauser }\end{array} & \text { https://www.youtube.com/watch?v=CuCiWjgpqSQ } \\ 11 & \text { El florido pensil } & \text { https://www.youtube.com/watch?v=eWqpgiokb0Y } \\ 12 & \text { Escuela de rock } & \text { https://www.youtube.com/watch?v=Ux7F8dYYK1A } \\ 13 & \text { Los chicos del coro } & \text { https://vimeo.com/36841265 } \\ 14 & \text { Matilda } & \text { https://www.youtube.com/watch?v=-knyam2y_v4 } \\ 15 & \text { Qué verde era mi valle } & \text { https://www.youtube.com/watch?v=bm6nPOboAx4 } \\ 16 & \text { Valentina } & \text { https://www.youtube.com/watch?v=iUo2ZOPcT2c }\end{array}$

\subsection{Escenas que muestran un contexto social}

$\begin{array}{lll}17 & 2+2=5 & \text { https://www.youtube.com/watch?v=MjPQUJTyXFQ } \\ 18 & \text { Binta y la gran idea } & \text { https://www.youtube.com/watch?v=rXDRYX8ECgA } \\ 19 & \text { Brubaker } & \text { https://vimeo.com/15523013 } \\ 20 & \text { Con ganas de triunfar } & \text { https://www.youtube.com/watch?v=9c-Gf7sDoMk } \\ 21 & \text { Espías desde el cielo } & \text { https://vimeo.com/177527408 https://vimeo.com/177527423 } \\ 22 & \text { If } & \text { https://www.youtube.com/watch?v=_haMUQH1zLc } \\ 23 & \text { La vida es bella } & \text { https://www.youtube.com/watch?v=49pjtXcFDlk } \\ 24 & \text { Sufragistas } & \text { https://www.youtube.com/watch?v=-E14JHfxu2I }\end{array}$

BLOQUE 2. NÚMEROS

\subsection{Números naturales}

25

26

27

28

29

30

31

32

33

34
Descifrando Enigma (15:20-16:30)

El Chavo del 8

El hombre que copiaba

El número 23

Futurama: El bocinazo T2 C18 (4:20-5:00)

La habitación de Fermat

La habitación de Fermat

La habitación de Fermat

La teoría del bigbang

Los payasos de la tele
https://www.youtube.com/watch?v=3LVCS9bmOks

https://www.youtube.com/watch?v=bPz5JAyoX5g

https://www.youtube.com/watch?v=q7RSHm33MwM

https://vimeo.com/113779015

http://diskokosmiko.mx/jml/futurama-28224/futurama-2x18-el-bocinazo-1999,230486.mkv

https://www.youtube.com/watch?v=jMTJPAY9B1Q https://www.youtube.com/watch?v=wzKJ2g213t0

https://www.youtube.com/watch?v=OaKJ8G81kek

https://www.youtube.com/watch?v=38HusD8K8lc

https://www.youtube.com/watch?v=ShkAMt0Ye2o

https://www.youtube.com/watch?v=R1k5KZNBf-M 


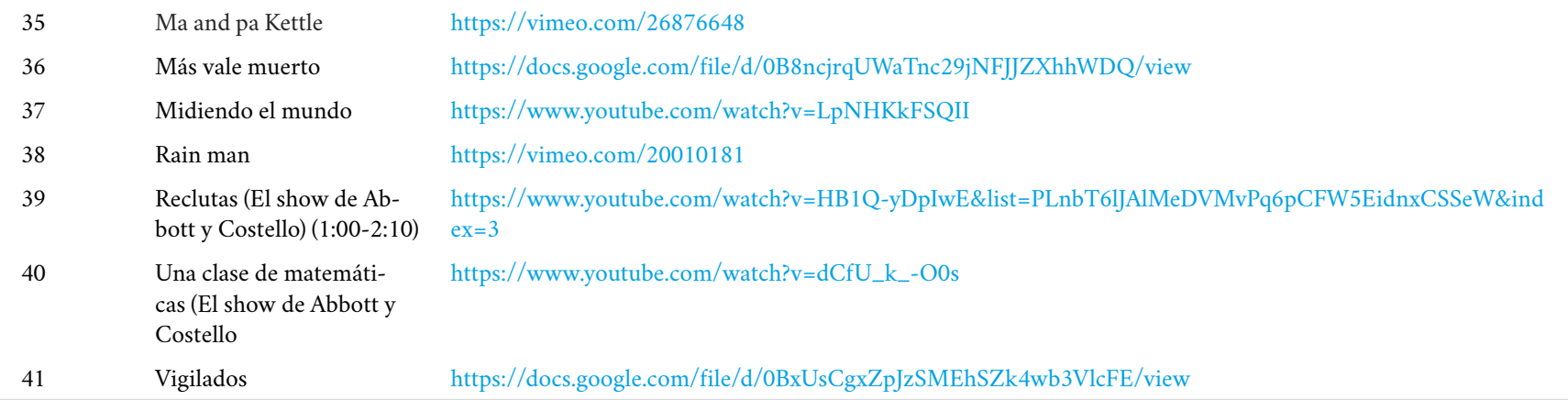

2.2. Números enteros
$42 \quad$ Con ganas de triunfar
https://www.youtube.com/watch?v=UTL4VR70NzQ
43 Los Simpson: T17 C19 (0:20-0:45)
https://www.youtube.com/watch?v=ocvEYALGV-s

\subsection{Números racionales, Fracciones}

$\begin{array}{lll}44 & \text { El virginiano } & \text { https://www.youtube.com/watch?v=BtmLHDuA-GA } \\ 45 & \text { Granujas de medio pelo } & \text { https://www.youtube.com/watch?v=0kp6EoBfzeU\&list=RD0kp6EoBfzeU\#t=0 } \\ 46 & \text { Los Simpson T9 C3 } & \mathrm{https://vimeo.com} / 27583813\end{array}$

\subsection{Números irracionales}

47 La fórmula preferida del https://www.youtube.com/watch?v=rFAF2ILSUF0 profesor (36:00-38:00)
$48 \quad$ La vida de Pi
https://vimeo.com/96178668
49 Los Simpson T12 C16
https://www.youtube.com/watch?v=0bX_bhwksjE
50 Noche en el museo 2
https://docs.google.com/file/d/0BxUsCgxZpJzSTkFwbWpHdVlfelU/view
$51 \quad$ Vigilados
https://www.youtube.com/watch?v=RAF5z_Gk_4M

\subsection{Potencias y raíces}

\section{2}

$1: 48)$

53

54

55

56

0:45)

$57 \quad$ Los Simpson T7 C6

$58 \quad$ Numb3rs

59 Súper Mario Bros

\subsection{Múltiplos y divisores}

60

61

62

63

64

65

66

Contact

Cube

El asesino del calendario

La fórmula preferida del profesor (11:00-13:20)

La fórmula preferida del profesor(24:40-27:03)

La habitación de Fermat

La soledad de los $n^{\text {os }}$ pri$\operatorname{mos}(6: 20-7: 00)$ https://vimeo.com/64054220

https://www.youtube.com/watch?v=h-BUti-DNFE

https://www.youtube.com/watch?v=9FZMFN2G1RI

https://www.youtube.com/watch?v=H2AqjCOeK-w

https://www.youtube.com/watch?v=o3kaSOAvKBM

https://vimeo.com/18750366

http://www.dailymotion.com/video/xem33t_estafa-piramidal_shortfilms

https://vimeo.com/68646901
https://www.youtube.com/watch?v=VnhvLXTryJs

https://vimeo.com/17972256

https://vimeo.com/18094321

https://www.youtube.com/watch?v=rFAF2ILSUF0

https://www.youtube.com/watch?v=rFAF2lLSUF0

https://www.youtube.com/watch?v=GjElx-fZX8s

https://www.youtube.com/watch?v=BYlnjLt9Htk 


\subsection{Proporcionalidad y porcentajes}

$\begin{array}{lll}67 & \text { 21 Blac jack } & \text { https://www.youtube.com/watch?v=S8NMSLCDlcY } \\ 68 & \text { Chicas malas (2:30-3:00) } & \text { https://www.youtube.com/watch?v=NWvyooVDwb0 } \\ 69 & \text { Con ganas de triunfar } & \text { https://www.youtube.com/watch?v=ctel7Bpo_gM } \\ 70 & \text { El bazar de las sorpresas } & \text { https://vimeo.com/152013993 } \\ 71 & \text { El mundo está loco, loco, } & \text { https://docs.google.com/file/d/0B8ncjrqUWaTnejZ5NmJqeWhMbDg/view } \\ & \text { loco } & \\ 72 & \text { Entrenador Carter } & \text { https://vimeo.com/70243376 } \\ 73 & \text { Vacaciones en Roma } & \text { https://vimeo.com/25357366 }\end{array}$

\subsection{Series y sucesiones}

$74 \quad$ Abducidos

75 La habitación de Fermat

$76 \quad$ Numb3rs

$77 \quad$ Pi, fe en el caos
https://www.youtube.com/watch?v=1KMmW_s66DI

https://vimeo.com/30630048

https://vimeo.com/18117981

https://www.youtube.com/watch?v=y0sfBfapWVI

\subsection{Otros}

78

79 Con ganas de triunfar

80 Smilla, misterio en la nieve

$81 \quad$ Toy Story

C5 (14:00-15:20)

Toy Story
Aquí no hay quien viva T2 https://www.youtube.com/watch?v=_jZ8XMp7JII
https://www.youtube.com/watch?v=xevoYXfY1BE

https://vimeo.com/14702010

https://www.youtube.com/watch?v=nNLfL7d2k-8

\section{BLOQUE 3: MEDIDA}

\subsection{Longitud y superficie}

82

83

\section{Los Simpson T7 C6}

Mister Bean C8
https://www.youtube.com/watch?v=xShqwOmXPCo

https://www.youtube.com/watch?v=4061Y7IYA6g\&sns=em

\subsection{Masa y peso}

$84 \quad$ Yo hice a Roque III

https://www.youtube.com/watch?feature=player_embedded\&v=wkJrysJhU7s

\subsection{Capacidad y volumen}

$\begin{array}{lll}84 & \text { La jungla de cristal } 3 & \text { https://www.youtube.com/watch?v=kSq42nPjnE0 } \\ 85 & \text { La jungla de cristal } 3 & \text { http://www.dailymotion.com/video/xbcehl_el-problema-de-los-bidones_tech }\end{array}$

3.4. Tiempo

$87 \quad$ Factotum (15:50-17:14) https://www.youtube.com/watch?v=MyqbdwZZ40Q

$88 \quad$ Sal gorda

89 Willy Fog C1

https://www.youtube.com/watch?v=2yKtGZov338

https://www.youtube.com/watch?v=GJUnJcKjeyA

\subsection{Otros}

90 Aquí no hay quien viva T2 https://www.youtube.com/watch? $v=\_j Z 8 X M p 7 J 1 I$

C5 (6:10-6:55, 25-25:50,

56-56:15)

91 Los Serrano (28:30-30:50) http://www.mitele.es/series-online/los-serrano/57a5a7dfc815da6b598b45e6/player

\section{BLOQUE 4: GEOMETRIA}

\subsection{Geometría plana}

$\begin{array}{lll}92 & \text { Adiós muchachos } & \text { https://vimeo.com/28243563 } \\ 93 & \text { El mago de Oz } & \text { https://www.youtube.com/watch?v=cCkowyTBrps } \\ 94 & \begin{array}{l}\text { El príncipe y el mendigo } \\ (4: 10-5: 00)\end{array} & \text { https://www.youtube.com/watch?v=K039BlG-nhA (4:10-5:00) } \\ & \text { La clase } & \text { https://vimeo.com/37206179 }\end{array}$




\begin{tabular}{|c|c|c|}
\hline 96 & $\begin{array}{l}\text { La fórmula preferida del } \\
\text { profesor }(0: 30-0: 45)\end{array}$ & https://www.youtube.com/watch?v=rFAF2ILSUF0 (0:30-0:45) \\
\hline 97 & $\begin{array}{l}\text { La soledad de los } \mathrm{n}^{\text {os }} \\
\text { primos. }\end{array}$ & https://vimeo.com/82083523 \\
\hline 98 & Las diabólicas & https://matedecine.wordpress.com/2011/06/22/las-diabolicas-el-area-del-hexagono/ \\
\hline 99 & $\begin{array}{l}\text { Los Simpson T5 C19 } \\
(1: 30-2: 20)\end{array}$ & https://www.youtube.com/watch?v=Xjz4f3Gbhek \\
\hline 100 & Misión imposible III & https://www.youtube.com/watch?v=1648Q-SDAM4 \\
\hline 101 & Tom y Jerry & https://vimeo.com/163902480 \\
\hline \multicolumn{3}{|c|}{ 4.2. Geometría especial } \\
\hline 102 & Ágora & https://vimeo.com/22848858 \\
\hline 103 & Los Simpson: T17 C19 & https://www.youtube.com/watch?v=8P90AEMrIKs \\
\hline \multicolumn{3}{|l|}{ 4.3. Otros } \\
\hline 104 & Annápolis (18:10-18:50) & https://www.youtube.com/watch?v=cQsRNXg3ahQ \\
\hline 105 & Águila roja T3, C37 & https://vimeo.com/106215361 \\
\hline 106 & Cortina rasgada & http://www.dailymotion.com/video/xpz5dp_pi_tech \\
\hline 107 & El coche fantástico & https://vimeo.com/35698978 \\
\hline 108 & Los Simpson: T7 C6 & https://vimeo.com/18750366 \\
\hline 109 & Möbius (Rochant) & https://vimeo.com/169801477 \\
\hline 110 & Moebius (Mosquera) & https://vimeo.com/21303855 \\
\hline 111 & Stargate & https://vimeo.com/18008143 \\
\hline 112 & $\begin{array}{l}\text { Tu nombre envenena mis } \\
\text { sueños }\end{array}$ & https://vimeo.com/19202706 \\
\hline 113 & Una mente maravillosa & https://vimeo.com/44431343 \\
\hline \multicolumn{3}{|c|}{ TEMA 5. ESTADÍSTICA Y PROBABILIDAD } \\
\hline \multicolumn{3}{|c|}{ 5.1. Estadística } \\
\hline 114 & Aida. C104 & https://drive.google.com/file/d/0B8UgVYW4VaOPVzRUbkptRVZHelE/view?pli=1 \\
\hline 115 & Ciudad mágica (5:50-7:00) & https://www.youtube.com/watch?v=GRpoTKLxev8 (5:50-7:00) \\
\hline \multicolumn{3}{|c|}{ 5.2. Probabilidad } \\
\hline 116 & 21 Blac jack & https://www.youtube.com/watch?v=uRxWQiuF3II \\
\hline 117 & Cuéntame cómo pasó & http://rtve.es/v/3924198 (01:00-1:32; 01:03:36-01:03:54; 1:08:39-01:09:00) \\
\hline 118 & El día de la bestia & http://www.dailymotion.com/video/xg6k9h_combinatoria-diabolica_tech \\
\hline 119 & El hombre que copiaba & https://www.youtube.com/watch?v=iKvB8Bw0YnU \\
\hline 120 & El puente sobre el río Kwai & https://vimeo.com/23755295 \\
\hline 121 & Intacto & https://vimeo.com/23477090 \\
\hline 122 & Justi\&cía $(49: 20-50: 30)$ & https://www.youtube.com/watch?v=oaaKMe0ZY7Q \\
\hline 123 & Numb3rs & https://www.youtube.com/watch?v=pqJBTWoIkbA \\
\hline \multicolumn{3}{|l|}{ 5.3. Otros } \\
\hline 124 & Los Simpson: T12 C9 & https://www.youtube.com/watch?v=ctR0-NDB7T4 \\
\hline 125 & Pandorum & https://www.youtube.com/watch?v=nmxFMZX5g60 \\
\hline
\end{tabular}




\title{
INFORMACIÓN SOBRE EL AUTOR
}

\begin{abstract}
Alberto Zapatera. Es Licenciado en Matemáticas, Diploma de Estudios Avanzados y Doctor en Didáctica de las Matemáticas. Actualmente profesor en la Universidad Cardenal Herrera CEU en Elche. Sus líneas de investigación se centran en el desarrollo profesional del profesor de matemáticas, metodologías innovadoras en el aprendizaje de las matemáticas y pensamiento algebraico. Tiene publicaciones en revistas nacionales (Revista Complutense de Educación, Revista de Didáctica de las Matemáticas UNO...) e internacionales (Journal of Mathematics Teacher Education, BOLEMA). Asimismo suele presentar comunicaciones en congresos nacionales e internacionales de investigación e innovación en el área de la Didáctica de las Matemáticas.
\end{abstract}

